\title{
Multiple Approaches to Numerical Modelling of Container Ship Squat in Confined Water
}

\author{
Zhen Kok ${ }^{a *}$, Jonathan Duffy ${ }^{b}$, Shuhong Chai ${ }^{c}$ and Yuting Jin ${ }^{d}$ \\ a National Centre for Maritime Engineering and Hydrodynamics, Australian Maritime College, \\ University of Tasmania, Launceston, TAS, 7250, Australia \\ zhen.kok@utas.edu.au \\ b National Centre for Maritime Engineering and Hydrodynamics, Australian Maritime College, \\ University of Tasmania, Launceston, TAS, 7250, Australia \\ j.duffy@utas.edu.au \\ c National Centre for Maritime Engineering and Hydrodynamics, Australian Maritime College, \\ University of Tasmania, Launceston, TAS, 7250, Australia \\ shuhong.chai@utas.edu.au \\ d Technology Centre for Offshore and Marine Singapore, 12 Prince George's Park, Singapore, \\ 118411, Singapore \\ jin_yuting@tcoms.sg
}

${ }^{*}$ Corresponding author:

\section{Zhen Kok}

National Centre for Maritime Engineering and Hydrodynamics

Australian Maritime College, University of Tasmania

Locked Bag 1395, Launceston, Tasmania, 7250

T: +61 401649847 | E: zhen.kok@utas.edu.au 


\section{Abstract}

Various URANS modelling techniques to predict container ship squat in confined water are investigated and compared in this study to assess the suitability of each modelling technique. Five methods are compared, among which three are quasi-statical estimations of squat from CFD computed hydrodynamic forces and moment (QS), and two are based on directly computed squat utilising dynamic overset meshing (OV) technique. In addition, the effect of self-propulsion on squat is investigated by comparing different methods of propulsion i.e. the hull is either towed (T) or selfpropelled by means of body-force propulsion virtual disc model (VD) or a fully discretised propeller (DP). The investigation shows that the QS methods tend to be superior in terms of computation efficiency, range of applicability and trim prediction accuracy. It is also shown that the effect of selfpropulsion is significant and should be accounted for to provide accurate results, especially at relatively high speeds. Moreover, virtual disc modelling is more computationally economical while also providing similar degree of accuracy to that of a discretised propeller. Thus, the most suitable method is the quasistatic method with virtual disc self-propulsion (QS-VD). However, for very shallow cases where $h / T<$ 1.14, the towed quasi-static squat model (QS-T) is recommended due to better accuracy.

Keywords: ship squat, discretised propeller, Reynolds Averaged Navier-Stokes equations, selfpropulsion 


\section{Nomenclature}

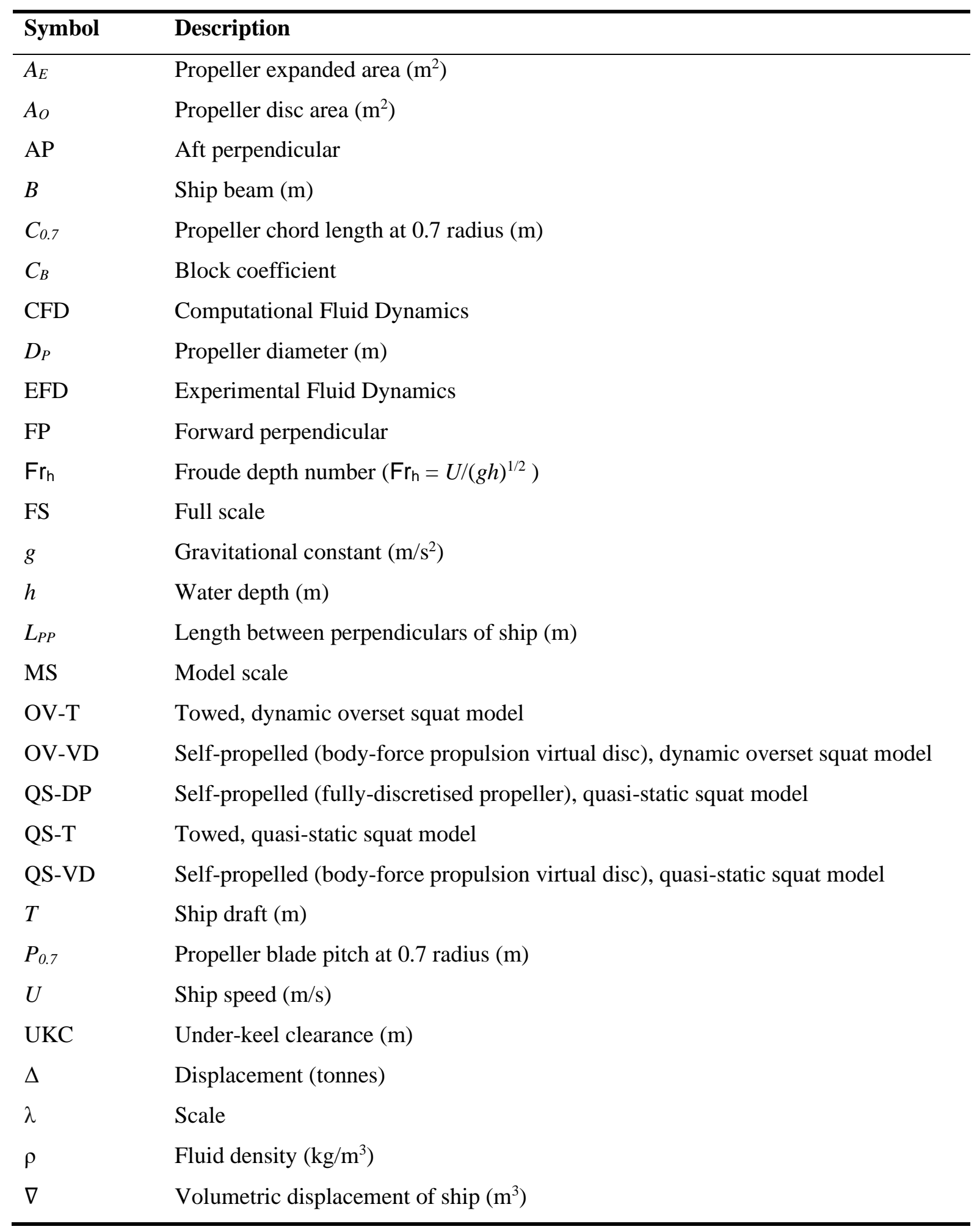




\section{$3 \quad 1$ Introduction}

4 In order to satisfy the increasingly competitive demands of the shipping industry, shipping operators

5 have turned to the use of larger ships. Also due to the more favourable economic return, the trend of

6 operating progressively larger ships is likely to continue in the future. However, dredging and harbour

7 expansion projects to accommodate larger ships are often unfavourable and are thus inevitably outpaced

8 by the size growth of next generation ships (Gourlay et al., 2015). Consequently, hydrodynamic

9 phenomena in confined waters, particularly ship squat, have and will continue to be severe threats to operational safety in ports and approach channels.

11 Ship squat in is one of the most prominent hydrodynamic issues in shallow waters and has received attention from researchers in the past. Constantine (1960) conducted pioneering investigations into the ship squat phenomena in confined waters where the different squat behaviours for subcritical $\left(\mathrm{Fr}_{\mathrm{h}}<1\right)$, critical $\left(F r_{h}=1\right)$ and supercritical $\left(F r_{h}>1\right)$ vessel speeds were discussed. Tuck (1966) formulated a slender-body theory that was valid for squat estimation in shallow water without lateral restrictions. The above theory was eventually modified to account for finite channel widths (Tuck, 1973). Beck et al. (1974) then extended Tuck's theory to dredged channels. Tuck's theory was further improved by Naghdi and Rubin (1984) where a nonlinear steady-state solution of the differential equations of the thin ship theory was implemented while Cong and Hsiung (1991) merged the flat ship and thin ship theory to enable the application of the method for transom stern ships.

Besides theoretical methods, extensive experiment-based prediction techniques have also been developed. However, it should be noted that most empirical techniques were developed based on full form ships and fewer methods relate directly to the more slender container ships. Dand and Ferguson (1973) presented squat measurements from model scale experiments and then developed a semiempirical prediction technique to predict squat for full form ships with reasonable accuracy. Fuehrer and Römisch (1977) used model tests to develop empirical formulae which account for different cross section parameters of the canal. Barrass (1979) also presented ship squat formulae based on model scale experiments accounting for ship speed, block coefficient and blockage factor. The effect of propulsion 
on ship squat in shallow water was investigated by Duffy and Renilson (2000) via model scale experiments where empirical corrections for the propulsion effect were presented for a bulk carrier hull form. Duffy (2008) presented a mathematical model to predict unsteady squat and dynamic acceleration effects for a ship traversing in non-uniform water depth. Delefortrie et al. (2010) developed a mathematical model with experimental data input to include the effects of muddy bottom and propeller action. Lataire et al. (2012) presented an improved semi-empirical model that considers the distribution of the cross-sectional areas of the ship as well as the longitudinal distribution waterline beam. Eloot et al. (2008) incorporated various experimental data of ship, environmental, operational and shipping traffic parameters to develop a Tuck-parameter based mathematical model. However, these investigations are all based on model scale experiments and have no corrections for scale effect.

With the advancement of computational power, numerical methods have become more viable and popular in the study of ship squat. Yao and Zou (2010) implemented a first-order 3D panel method to predict the sinkage and trim of a ship travelling in shallow water where the method was found to be able to predict sinkage and trim with satisfactory accuracy for subcritical and supercritical speeds, but not for trans critical speeds as non-linear effects were neglected by the method. Zhang et al. (2015) developed a slender body theory based potential flow solution to investigate the hydrodynamic pressure field around a ship travelling in shallow open water, rectangular canal, dredged channel and stepped canal where the method was found to be validated against experimental results in different waterways, but again, the method is unsuitable for conditions near the critical speed and above. Gourlay et al. (2016) compared the estimated ship squat in confined water for a range of lateral widths and water depths computed from four different potential flow methods; linear 2D, non-linear 1D, double body and a Rankine source code. However, the four methods each have their disadvantages such as inaccuracy at high speeds, limited canal width range applicability and inability to resolve short wavelengths at low speeds, respectively.

Besides potential flow based methods, the computational fluid dynamics (CFD) method has also been adopted in the study of ship squat where non-linear and viscous effects can be modelled. Jachowski 
(2008) conducted investigations into ship squat in shallow water using a commercial ReynoldsAveraged Navier-Stokes (RANS) solver and showed that the CFD results agree well with empirical methods, experimental observations and wave theory; however, further investigation is necessary to model the effects of lateral restriction and channel bottom irregularity. Shevchuk et al. (2016) conducted both RANS and RANS/Large Eddy Simulation (LES) for comparison and to identify the cause of squat intensification at $h / T<1.3$, which is often observed in experiments. The study concluded that the intensification is due to the union of boundary layers developed both from the ship hull and channel bottom, and that the nature of the RANS model is unable to capture fully flow separation at the stern; however this drawback does not cause noticeable changes to the prediction accuracy. Tezdogan et al. (2016) performed an unsteady RANS simulation to predict the squat and resistance of a ship appended with a fixed propeller in constrained water conditions where the midship sinkage was predicted accurately against experimental results but trim was excluded from the analysis.

As mentioned earlier, container ship size is steadily outpacing channel dredging rate. Hence, there is a need to more accurately predict container ship squat to maximise cargo delivery whilst avoiding grounding. In addition, existing prediction techniques are mostly concerned with the lower under-keel clearance (UKC) conditions and therefore lower speeds. Consequently, there is uncertainty with respect to the accuracy of these techniques at higher speeds for deeper UKC conditions. Kok et al. ("Comparison of of URANS Prediction of Self-Propelled Container Ship Squat against Empirical Methods and Benchmark Data", submitted, International Journal of Maritime Engineering, RINA, London, UK) have also shown that most empirical formulae are inaccurate for $h / T>1.14$ at $\mathrm{Fr}_{\mathrm{h}}>0.5$, whereas the self-propelled URANS CFD squat simulation presented in that study demonstrated good correlation with benchmark data for the said conditions.

Thus, the aim of the present work is to assess the suitability of different CFD modelling techniques in the prediction of container ship squat in shallow water. The investigation was conducted using a commercial unsteady RANS solver, STAR-CCM+ with consideration of propeller effect and free surface effect. The findings of the present work serve to determine the most suitable modelling 
technique for further systematic studies particularly regarding scale effect which has been highlighted to be insufficiently addressed. The ultimate aim of future work will be to produce a suitable prediction technique that is valid for full scale cases and a wide range of $h / T$ and ship speeds which are based on a series of systematic studies using the modelling technique presented in this study.

\section{Hull Form and Tank Geometry}

Simulations were set-up to match one of the PreSquat workshop benchmark cases conducted in the Federal Waterways Engineering and Research Institute (BAW) where the Duisburg Test Case (DTC) hull appended with a propeller operating in an asymmetrical canal were investigated at a 1:40 scale (Mucha et al., 2014). The DTC is a generic 14,000 TEU container ship for benchmarking purposes developed by the Institute of Ship Technology, Ocean Engineering and Transport Systems (ISMT) of the University of Duisburg-Essen. The propeller used in the experiments in the PreSquat workshop was the Wageningen B-series 4-bladed propeller operated at model self-propulsion point. Figure 1 depicts the profile view of the DTC hull with the body plan and the Wageningen B-series propeller. Table 1 summarises the principal particulars of the hull and propeller. A cross section view of the asymmetric canal and the corresponding full scale dimensions are shown in Figure 2.
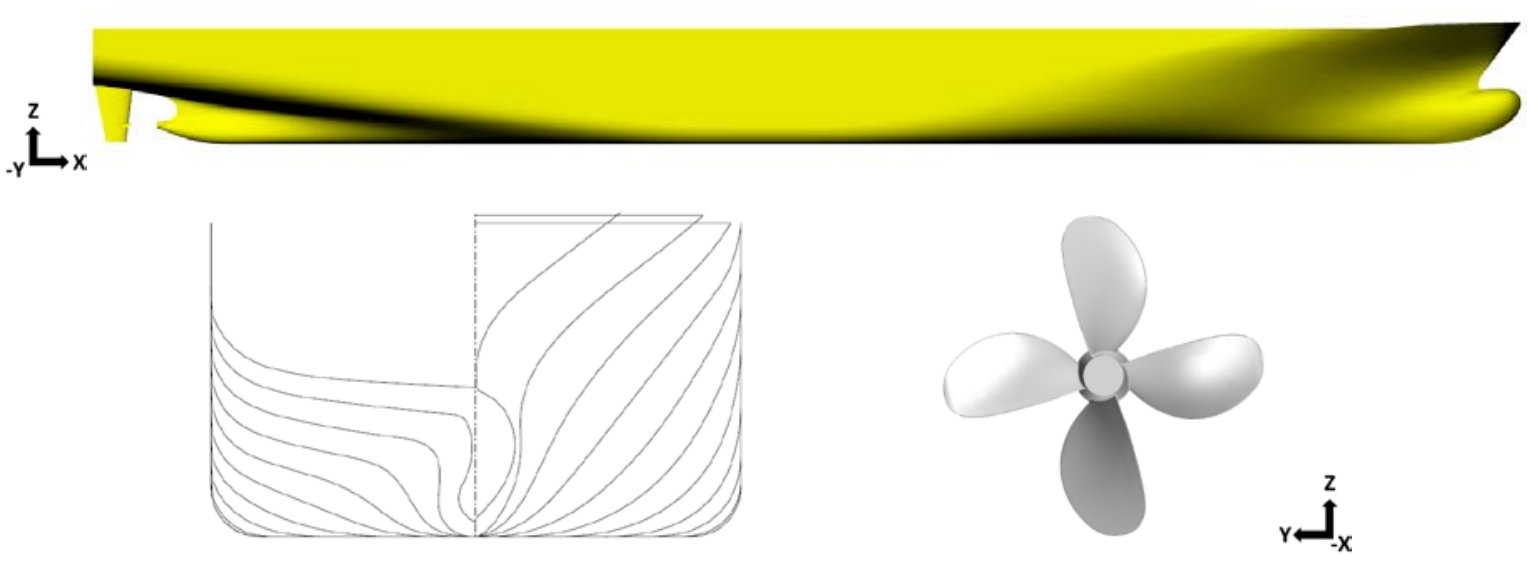

Figure 1: Profile view of the DTC hull (top), the body plan (bottom left) and the Wageningen B-series propeller investigated. 
Table 1: Principal particulars of the DTC hull and the Wageningen B-series propeller.

\begin{tabular}{|l|l|l|}
\hline Principal Particulars & Model Scale (1:40) & Full Scale (1:1) \\
\hline \multicolumn{3}{|c|}{ Ship Particulars } \\
\hline$L_{P P}(\mathrm{~m})$ & 8.875 & 355 \\
\hline$B(\mathrm{~m})$ & 1.275 & 51.0 \\
\hline$T(\mathrm{~m})$ & 0.350 & 14.0 \\
\hline$\Delta$ (tonnes) & 2.618 & 163.5 \\
\hline$C_{B}$ & 0.661 & 0.661 \\
\hline \multicolumn{2}{|l|}{ Propeller Particulars } \\
\hline$D_{P}(\mathrm{~m})$ & 0.223 & 8.92 \\
\hline Blades & 4 & 4 \\
\hline$P_{0.7} / D_{P}$ & 1.275 & 1.275 \\
\hline$A_{E} / A_{O}$ & 0.55 & 0.55 \\
\hline$C_{0.7}(\mathrm{~m})$ & 0.066 & 2.635 \\
\hline & &
\end{tabular}

101

102

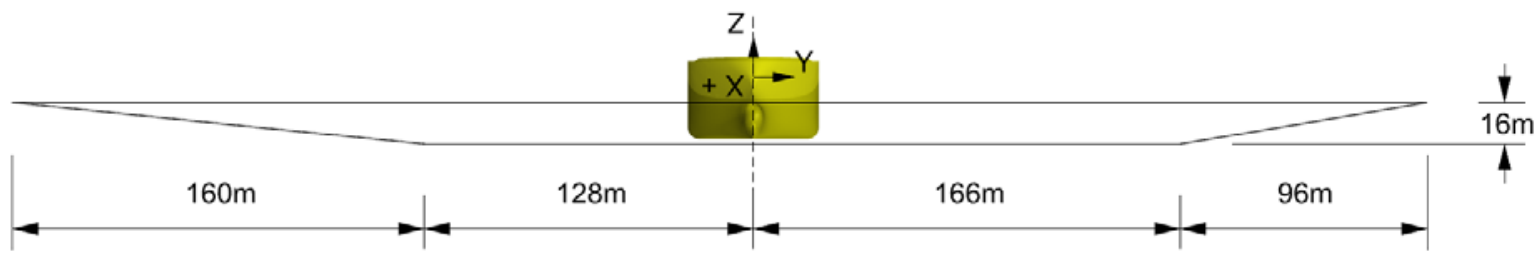

Figure 2: Cross section view of the asymmetric canal geometry.

\section{Computational Methods}

Five distinct modelling techniques were to be compared in this study, which include a towed quasistatic approach (QS-T), a self-propelled quasi-static approach by means of virtual disc modelling (QSVD), a self-propelled quasi-static approach by means of discretised propeller modelling (QS-DP), a towed dynamic overset approach (OV-T) and a self-propelled dynamic overset approach by means of virtual disc modelling (OV-VD). Note that despite the implementation of self-propulsion in the experiment, towed models were still included in the study for the purpose of quantifying the effect of self-propulsion and also for the purpose of assessing the accuracy in predictions without self-propulsion.

The QS techniques (i.e. QS-T, QS-VD and QS-DP) were designed such that the hull was constrained in all degrees of freedom except forward translation whereas the OV techniques (i.e. OV-T and OV- 
VD) allow the hull to translate forward, sink and trim. URANS simulations were performed to investigate the hydrodynamic forces and moments acting on the hull where the resultant trim and sinkage were then approximated for the case of the QS techniques using hydrostatic data for the hull.

Note that only one iteration of the force and moment balance was conducted as a previous study using only one iteration of this method has shown favourable results. However, this also implies that nonlinearities in forces and moments as the hull squats will not be resolved.

For the case of the OV techniques, the dynamic trim and sinkage were directly computed in the URANS simulations via the dynamic fluid body interaction (DFBI) module. The key differences among the five modelling techniques are summarised in Table 2. As summarised in Table 2, the QS-T and QS-VD methods do not have overset meshing but the QS-DP has overset mesh for the propeller while the OVT and OV-VD methods have overset mesh for the entire hull.

It should be noted, however, that the set-up and results obtained from the QS-DP method have been discussed in a previous investigation by the authors and is presented in this paper for comparison purposes only. The methods implemented for QS-DP is only briefly discussed in this paper and thus, reference should be made to the work presented by Kok et al. (2019) for further details. The remainder of this section discusses more on the methods of the other four techniques. Regardless, the commercial CFD solver STAR-CCM+ was used to conduct all the computations where the integral form of incompressible RANS equation is resolved using finite volume method of discretisation.

Table 2: Summary of key differences between the different CFD techniques to be compared.

\begin{tabular}{|l|l|l|l|l|l|}
\hline Technique Name & QS-T & QS-VD & QS-DP & OV-T & OV-VD \\
\hline Overset Mesh & Absent & Absent & $\begin{array}{l}\text { Present } \\
\text { (propeller) }\end{array}$ & $\begin{array}{l}\text { Present } \\
\text { (hull) }\end{array}$ & $\begin{array}{l}\text { Present } \\
\text { (hull) }\end{array}$ \\
\hline $\begin{array}{l}\text { Propulsion } \\
\text { Method }\end{array}$ & $\begin{array}{l}\text { Towed (no } \\
\text { propeller) }\end{array}$ & Virtual Disc & $\begin{array}{l}\text { Discretised } \\
\text { Propeller }\end{array}$ & $\begin{array}{l}\text { Towed (no } \\
\text { propeller) }\end{array}$ & Virtual Disc \\
\hline $\begin{array}{l}\text { Sinkage \& Trim } \\
\text { Calculation }\end{array}$ & $\begin{array}{l}\text { Hydrostatic } \\
\text { Data }\end{array}$ & $\begin{array}{l}\text { Hydrostatic } \\
\text { Data }\end{array}$ & $\begin{array}{l}\text { Hydrostatic } \\
\text { Data }\end{array}$ & $\begin{array}{l}\text { DFBI Trim } \\
\text { \& Sinkage }\end{array}$ & $\begin{array}{l}\text { DFBI Trim } \\
\text { \& Sinkage }\end{array}$ \\
\hline
\end{tabular}


134

135

136

137

138

139

$140 \quad \frac{\partial \mathbf{U}_{\mathbf{i}}}{\partial \mathbf{x}_{\mathbf{i}}}=0$

$141 \rho \frac{\partial \boldsymbol{U}_{\boldsymbol{i}}}{\partial t}+\rho \frac{\partial}{\partial \boldsymbol{x}_{j}}\left(\boldsymbol{U}_{\boldsymbol{i}} \boldsymbol{U}_{j}\right)=-\frac{\partial \boldsymbol{P}}{\partial \boldsymbol{x}_{\boldsymbol{i}}}+\frac{\partial}{\partial \boldsymbol{x}_{\boldsymbol{j}}}\left(2 \mu \boldsymbol{S}_{i j}-\rho \overline{\boldsymbol{u}_{i}^{\prime} \boldsymbol{u}_{j}^{\prime}}\right)$

$142 \quad \boldsymbol{S}_{i j}=\frac{1}{2}\left(\frac{\partial \boldsymbol{U}_{i}}{\partial \boldsymbol{x}_{j}}+\frac{\partial \boldsymbol{U}_{j}}{\partial \boldsymbol{x}_{i}}\right)$

\subsection{RANS Equations} 2014): following section.

\subsection{Physics Modelling} to the $k$-omega $(k-\omega)$ model (Tezdogan et al., 2016).

It should be noted that field properties become random functions of space and time in turbulent flow. Hence, to resolve this, the velocity and pressure fields can be expressed as the sum of mean and fluctuating parts. By applying these mean and fluctuating parts into the incompressible form of NavierStokes equations, the Reynolds-averaged Navier-Stokes equations are effectively derived (CD-Adapco,

In the equations above, spatial indexes are represented as $i$ and $j$, while the time-averaged velocity and pressure fields are $\boldsymbol{U}_{i(i)}$ and $\boldsymbol{P}$ respectively. $\rho$ and $\mu$ represent the density of the effective flow and viscosity respectively. The term $S_{i j}$ is the mean strain-rate tensor whereas $\overline{\boldsymbol{u}_{i}^{\prime} \boldsymbol{u}_{j}^{\prime}}$ is the Reynolds stress tensor which is sometimes expressed as $\boldsymbol{\tau}_{\mathbf{i j}}$. The Reynolds stress tensor is symmetric and therefore possesses six components. Nonetheless, the Reynolds stress tensor remains unknown since three more unknown quantities are introduced into the equations when the instantaneous properties are decomposed into mean and fluctuating parts. Consequently, additional equations (turbulence models) are required to close the system. The closure of the equations for this particular investigation is discussed in the

In this investigation, the closure of the RANS equations was achieved by implementing the standard $k$ epsilon $(k-\varepsilon)$ turbulence model for all modelling techniques as sinkage prediction is not sensitive to turbulence models (Deng et al., 2014) and the $k$ - $\varepsilon$ model is also computationally economical compared 
According to Deng et al. (2014), the sinkage experienced by a hull as it advances is dependent on the free surface position and this is especially true for confined water conditions. Therefore, modelling of the free surface has been taken into account in this investigation by applying the volume of fluid (VOF) method. The second order discretisation scheme was applied to obtain sharp interfaces between water and air.

In order to simulate the rigid body hull motion as it was being towed or self-propelled, the DFBI setting was enabled on the hull. For the case of the QS-T and QS-VD methods, DFBI trim and sinkage was disabled to record the experienced hydrodynamic forces and moments for subsequent manual computation of the resultant trim and sinkage using basic hydrostatic data for the hull. In contrast, for the case of the OV-T and OV-VD methods, dynamic trim and sinkage were enabled where the governing equations of rigid body motions were solved.

A virtual disc model was also implemented in the solver to model the propeller but only for the QS-VD and OV-VD investigations. The body force propeller method was applied in the virtual disc model as this method models the flow field interaction between the hull and the propeller. Thus, the body force propeller method can simulate propeller force while having a computational cost advantage over fully discretised propellers (CD-Adapco, 2014). The open water performance curve data for the propeller were supplemented to the virtual disc model for computation of the resultant propeller induced-flow characteristics based on the flow around the hull. In contrast, the QS-DP method has a fully discretised propeller where the propeller modelling has been validated in an open-water propeller simulation in a previous study (Kok et al., 2019).

\subsection{Computational Domain, Boundary Conditions and Mesh Development}

The design of the computation domain was made to match the experimental set-up for the benchmark case discussed earlier and therefore the position of the hull within the domain and the cross-section of the domain are identical to that shown in Figure 2 whereas the position of the outlet, inlet and top boundaries can be seen in Figure 3. 


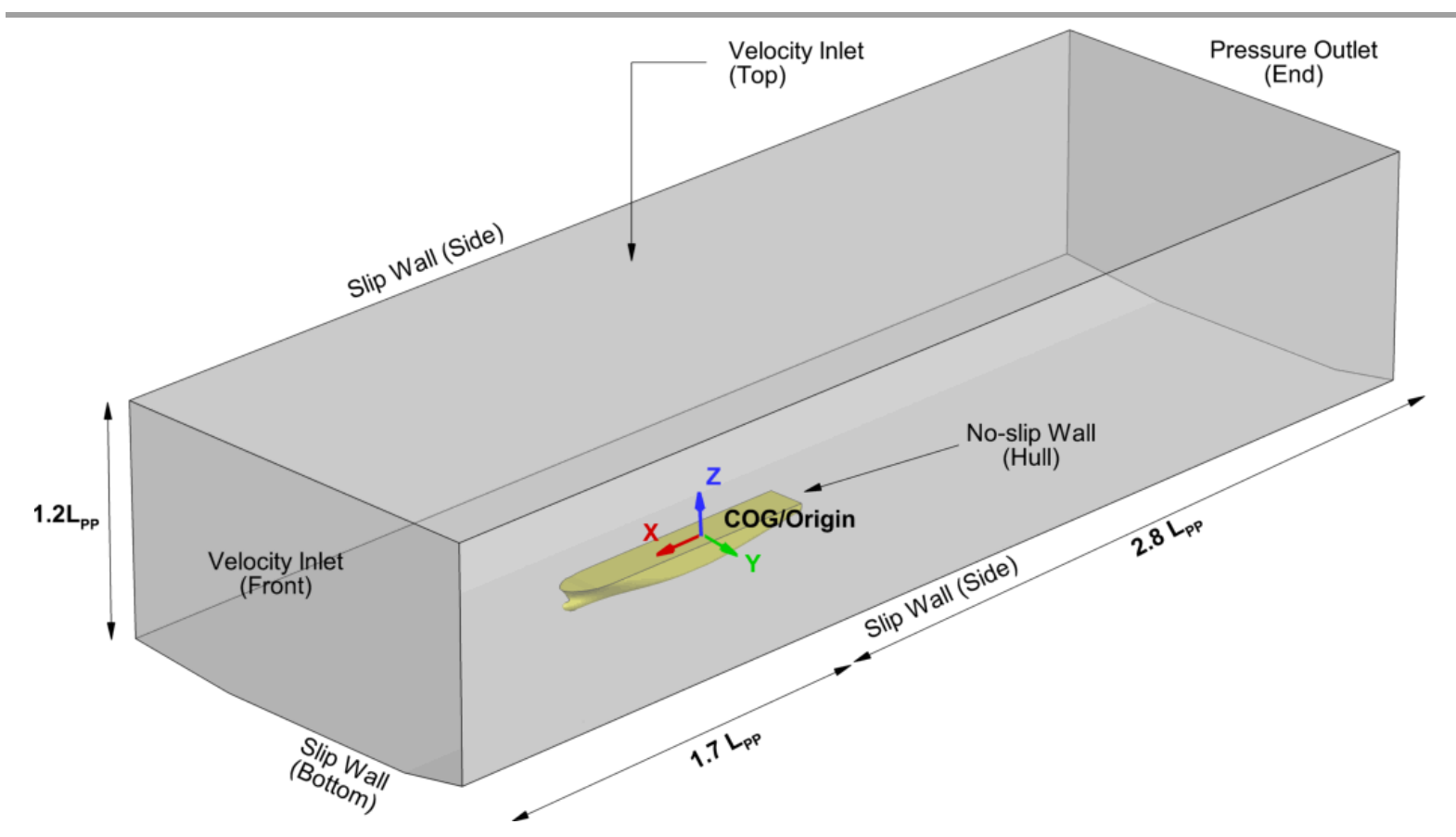

Figure 3: The domain dimensions and boundary conditions.

Zero velocity flat waves were generated at the inlet and backflow was prevented by the pressure outlet.

VOF wave damping of length $1.13 L_{P P}$ was also applied to the inlet and outlet to prevent unrealistic wave reflections from the said boundaries. The top of the domain was assigned as a zero velocity inlet.

No-slip wall condition was applied to the surfaces of the hull to capture the boundary layers that develop as the hull advances. However, the two side walls and bottom of the domain were assigned as slip walls because the set-up of the simulation is such that the domain moves together with the hull and therefore slip walls were required to prevent the development of a velocity profile due to the domain's motion. Nonetheless, in reality, boundary layers will eventually develop on the bottom floor beneath the hull and increase in thickness near the stern due to the induced flow velocity by the moving hull but the slipwall will be unable to capture this. Regardless, Deng et al. (2014) have shown that squat is insensitive to the near wall treatments applied to the bottom of the domain.

For all five methods, the computational grids were generated using the STAR-CCM+ built-in hexahedral trimmed cell mesher and surface remesher with reference to CD-Adapco (2014) recommendations for virtual towing tank simulations. Attention was given to provide additional mesh refinement to the hull surfaces, free surface region and the narrow underkeel clearance to accurately 
capture the developed boundary layers, Kelvin wave pattern and the underkeel flow characteristics respectively. Slow cell growth rate was applied to ensure a smooth mesh size transition between regions of highly refined mesh and the coarser mesh regions. The turbulent boundary layer was modelled using the prism layer mesher to achieve $y+$ value of 30 or above. Figure 4 depicts a perspective view of the generated computation domain mesh (similar for all five methods).

204 However, additional dynamic overset meshing is applied to the discretised propeller of the QS-DP meshing is applied to the entire hull of the OV methods to enable hull motion relative to the background.

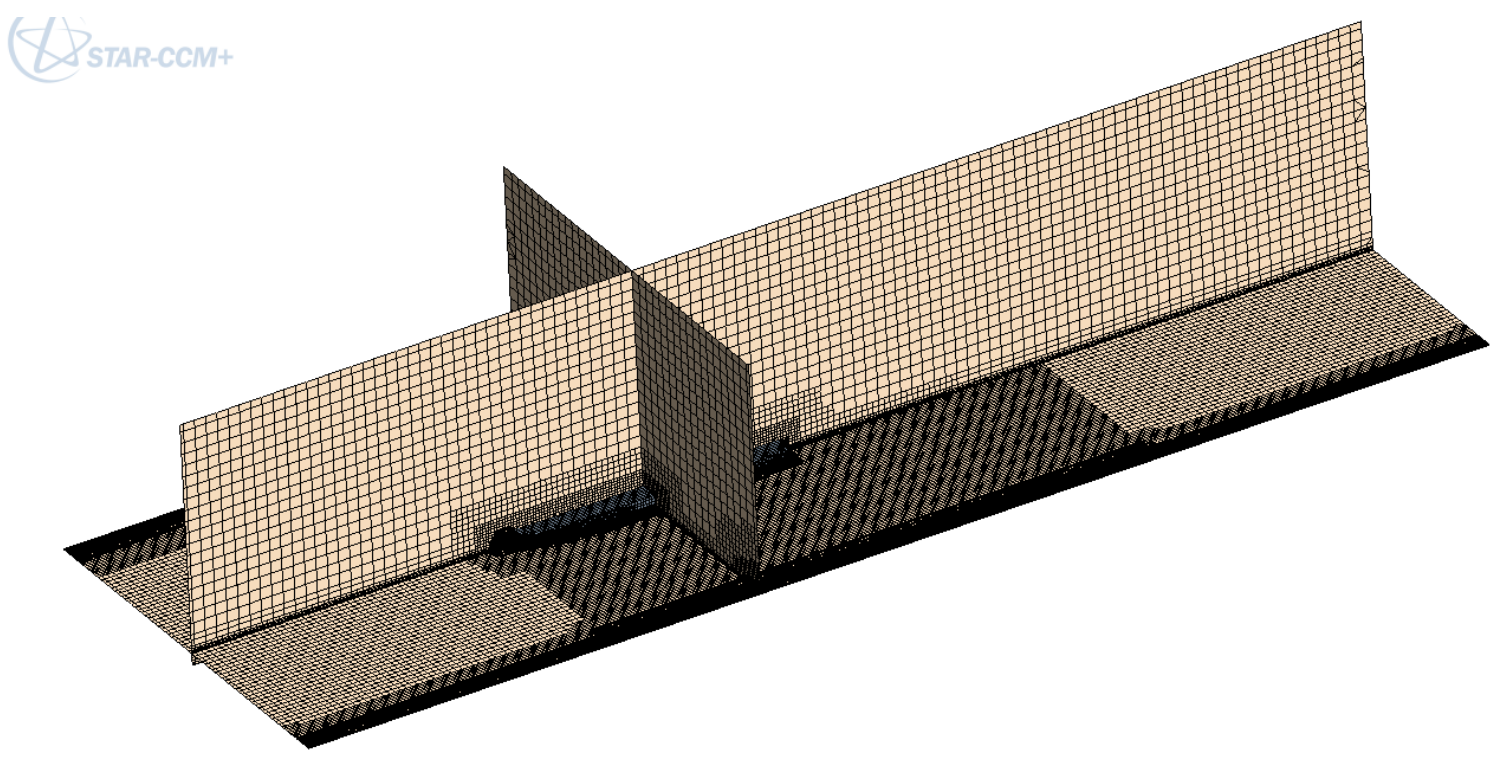

Figure 4: Perspective view of the mesh generated for the computation domain. 


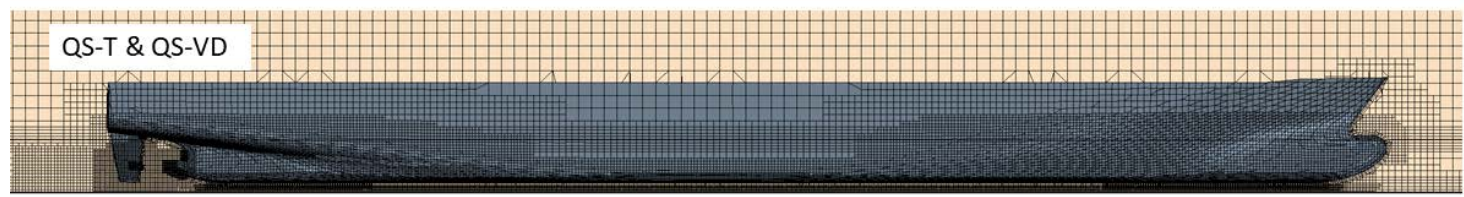

(a)

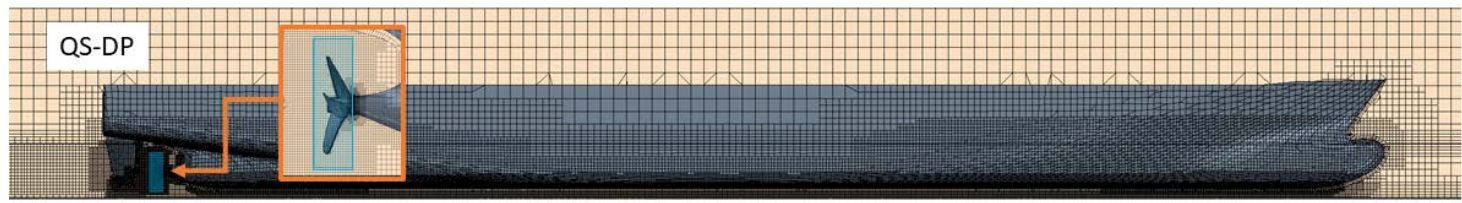

(b)

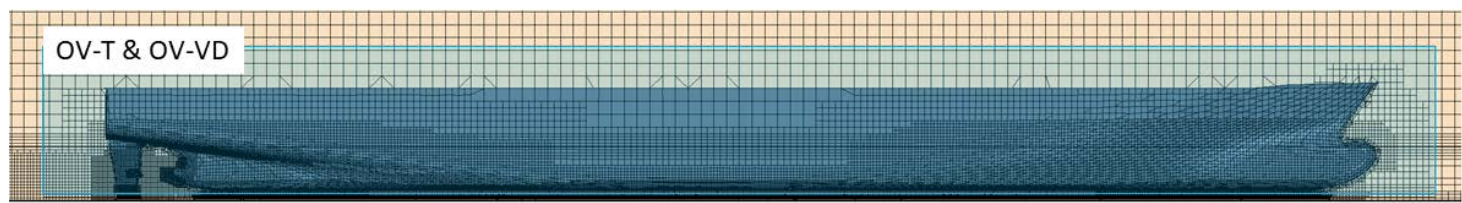

(c)

211 Figure 5: Profile view of the hull mesh for (a) QS-T and QS-VD methods, (b) the QS-DP method where overset mesh is applied to the discretised propeller, (c) the OV-T and OV-VD methods where overset mesh is applied to the hull.

\section{Verification and Validation}

215 In this investigation, verification and validation were conducted by application of the triplets method 216 discussed by Wilson et al. (2001) and Stern et al. (2001). The QS-VD method was implemented for the

217 verification and validation study because it is stable at high ship speeds unlike OV methods (further 218 discussions available in Section 6.1) and the degree of complexity of the QS-VD is representative of 219 the other methods. The case investigated was at $h / T=1.23$ where $\mathrm{Fr}_{\mathrm{h}}=0.553$.

\subsection{Verification and Numerical Uncertainty Analysis}

221 The numerical uncertainty $U_{S N}$ was approximated as the combination of iterative convergence uncertainty $U_{I}$, grid spacing uncertainty $U_{G}$ and time step uncertainty $U_{T}$ as shown in Equation 1 . Note

223 that $U_{I}$ was neglected as the iterative uncertainty for ship motion response simulations in STAR-CCM+

224 URANS solver is less than 0.2\% (Tezdogan et al., 2015). 
For both the grid and time step uncertainty convergence study, triple solutions were each obtained where the grid spacing uncertainty analysis was conducted with the smallest time-step while the time-step uncertainty study was conducted with the finest mesh setting. In the grid spacing uncertainty study, a refinement ratio $r_{G}=\sqrt{2}$ was applied to both the background and overset region. Details of the mesh count for the grid spacing uncertainty study are as shown in Table 3.

Table 3: Mesh count details in the grid spacing uncertainty study.

\begin{tabular}{|l|l|}
\hline Mesh Configuration & Total Mesh \\
\hline Coarse (3) & $1,355,800$ \\
\hline Medium (2) & $2,304,861$ \\
\hline Fine (1) & $3,388,145$ \\
\hline
\end{tabular}

In regards to the time-step uncertainty study, the time-step was determined using equation (2) where $\Delta l$ is the mesh dimension, $U$ is the ship speed and the Courant number CFL was set to value of 1 . The refinement ratio for time-step $r_{T}$ was 2 .

$$
\Delta t=\frac{\mathrm{CFL} \times \Delta l}{U}
$$

The changes in solution $\varepsilon$, between the three consecutive grids and three time steps were calculated where $S$ represents the solution obtained for that particular grid $\left(S_{G}\right)$ or time step $\left(S_{T}\right)$ :

$$
\begin{aligned}
& \varepsilon_{32}=S_{3}-S_{2} \\
& \varepsilon_{21}=S_{2}-S_{1}
\end{aligned}
$$

The convergence ratio $R_{i}$ was then determined based on the changes in solution using the following relation:

241

$$
R_{i}=\frac{\varepsilon_{21}}{\varepsilon_{32}}
$$

There are four possible outcomes when assessing the convergence ratio $R_{i}$ and they are:

1) $0<R_{i}<1$, where monotonic convergence has been achieved (MC)

2) $R_{i}<0 ;\left|R_{i}\right|<1$, where oscillatory convergence has been achieved (OC) 
3) $1<R_{i}$, where monotonic divergence has been achieved (MD)

4) $R_{i}<0 ;\left|R_{i}\right|>1$, oscillatory divergence has been achieved (OD)

The outcome of the grid spacing and time-step uncertainty analysis are as summarised in

Table 4 and Table 5, respectively. The verification study accounts for the AP and FP sinkage as well as the ship speed because the ship speed influences the squat experienced and the ship speed itself is also affected by the mesh quality and time-step. Observations on the change of ship speed, AP and FP sinkage solutions among the three grid spacings show monotonic convergence where the corresponding uncertainties are less than $10 \%$. Conversely, oscillatory convergence was achieved for time-step uncertainty of the ship speed, AP and FP sinkage where the uncertainties are less than $6 \%$. Therefore, the verification study conducted suggests that the computational model yields acceptable numerical uncertainties.

Table 4: Grid spacing uncertainty analysis summary.

\begin{tabular}{|c|c|c|c|c|c|c|c|}
\hline \multirow{2}{*}{ Variable } & \multirow[b]{2}{*}{$\mathbf{r}_{\mathbf{G}}$} & \multicolumn{3}{|c|}{ Solutions } & \multirow{2}{*}{$\mathbf{R}_{\mathbf{G}}$} & \multirow{2}{*}{ Convergence } & \multirow{2}{*}{$\begin{array}{l}U_{G} \\
\left(\% S_{G 1}\right)\end{array}$} \\
\hline & & $\mathrm{S}_{\mathrm{G} 3}$ & $\mathrm{~S}_{\mathrm{G} 2}$ & $\mathrm{~S}_{\mathrm{G} 1}$ & & & \\
\hline Ship Speed (m/s) & $\sqrt{2}$ & 0.5672 & 0.5545 & 0.5528 & 0.333 & $\mathrm{MC}$ & 1.02 \\
\hline AP Sinkage (m) & $\sqrt{2}$ & $4.75 \times 10^{-2}$ & $4.49 \times 10^{-2}$ & $4.39 \times 10^{-2}$ & 0.226 & $\mathrm{MC}$ & 8.66 \\
\hline FP Sinkage (m) & $\sqrt{2}$ & $2.51 \times 10^{-2}$ & $2.37 \times 10^{-2}$ & $2.33 \times 10^{-2}$ & 0.242 & MC & 4.58 \\
\hline
\end{tabular}

Table 5: Time-step uncertainty analysis summary.

\begin{tabular}{|c|c|c|c|c|c|c|c|}
\hline \multirow{2}{*}{ Variable } & \multirow[b]{2}{*}{$\mathbf{r}_{\mathbf{G}}$} & \multicolumn{3}{|c|}{ Solutions } & \multirow{2}{*}{$\mathbf{R}_{\mathbf{G}}$} & \multirow{2}{*}{ Convergence } & \multirow{2}{*}{$\begin{array}{l}\mathrm{U}_{\mathrm{G}} \\
\left(\% \mathrm{~S}_{\mathrm{T} 1}\right)\end{array}$} \\
\hline & & $\mathrm{S}_{\mathrm{T3}}$ & $\mathrm{S}_{\mathrm{T} 2}$ & $\mathrm{~S}_{\mathrm{T} 1}$ & & & \\
\hline Ship Speed $(\mathrm{m} / \mathrm{s})$ & 2 & 0.5631 & 0.5503 & 0.5528 & -0.20 & OC & 1.16 \\
\hline AP Sinkage (m) & 2 & $4.67 \times 10^{-2}$ & $4.21 \times 10^{-2}$ & $4.34 \times 10^{-2}$ & -0.41 & OC & 4.66 \\
\hline FP Sinkage (m) & 2 & $2.43 \times 10^{-2}$ & $2.21 \times 10^{-2}$ & $2.33 \times 10^{-2}$ & 0.242 & OC & 5.17 \\
\hline
\end{tabular}




\subsection{Validation Against Model Test Data}

Validation for the numerical method was conducted against experimental data by computing and comparing the comparison error, $E$, with the validation uncertainty, $U_{V}$, which is the combination of numerical uncertainty, $U_{S N}$, and experimental uncertainty, $U_{D}$, as given below:

$$
U_{V}=\sqrt{U_{S N}^{2}+U_{D}^{2}}
$$

The comparison error, $E$, is given as the difference between the experimental data, $D$, and simulation data, $S$. The numerical results are considered to be validated if $E$ is less significant than $U_{V}$ :

$$
E=D-S
$$

The value of $U_{D}$ was not provided in the literature referenced and was assumed to be within $5 \%$. $U_{V}$ was computed and then compared with $\mathrm{E}$ as depicted in Table 6. It can be seen that the estimated $\mathrm{E}$ for both AP and FP sinkage are clearly smaller that the estimated $U_{V}$ and are therefore validated. The result of this validation implies that the current numerical method is feasible for further simulations.

Table 6: Validation results.

\begin{tabular}{|l|l|l|l|l|}
\hline Sinkage & $\boldsymbol{U}_{S N}(\mathbf{\%})$ & $\boldsymbol{U}_{\boldsymbol{D}} \mathbf{( \% )}$ & $\boldsymbol{U}_{\boldsymbol{V}} \mathbf{( \% )}$ & $\boldsymbol{E} \mathbf{( \% )}$ \\
\hline AP & 6.91 & 5.00 & 8.53 & 5.93 \\
\hline FP & 9.84 & 5.00 & 11.03 & 9.00 \\
\hline
\end{tabular}

\section{Simulation Cases}

By adapting the verified and validated QS-VD set-up methodology to the other modelling techniques, a systematic study was conducted to compare and determine the most efficient and suitable modelling technique. The comparison is conducted at three static even-keel drafts each with varying speed ranges according to the benchmark data as tabulated in Table 7. The channel geometry for each case is given in Figure 2. Table 8 shows the resulting mesh count for each modelling technique at different $h / T$. 
Table 7: List of simulation cases.

\begin{tabular}{|l|l|l|l|l|}
\hline \multirow{2}{*}{$\begin{array}{l}\text { Full-Scale } \\
\text { Draft (m) }\end{array}$} & $\mathbf{h} / \mathbf{T}$ & \multicolumn{2}{|c|}{ Ship Speed } & \multirow{2}{*}{$\mathbf{r}_{\mathbf{h}}$} \\
\cline { 3 - 4 } & & Full Scale (knots) & Model Scale (m/s) & \\
\hline 13.0 & 1.23 & $5.06-13.56$ & $0.41-1.10$ & $0.21-0.56$ \\
\hline 14.0 & 1.14 & $4.83-13.23$ & $0.39-1.08$ & $0.20-0.54$ \\
\hline 14.5 & 1.10 & $2.42-12.20$ & $0.20-0.99$ & $0.10-0.50$ \\
\hline
\end{tabular}

Table 8: Mesh count for each modelling technique at varying $\mathrm{h} / \mathrm{T}$.

\begin{tabular}{|l|l|c|c|c|c|c|}
\hline h/T & Mesh Count & QS-T & QS-VD & QS-DP & OV-T & OV-VD \\
\hline 1.23 & Overset & - & - & 308,390 & $1,045,536$ & $1,076,902$ \\
& Background & - & - & $3,679,945$ & $2,678,573$ & $2,722,309$ \\
& Total & $\mathbf{3 , 3 5 0 , 6 8 8}$ & $\mathbf{3 , 3 8 8 , 1 4 5}$ & $\mathbf{3 , 9 8 8 , 3 3 5}$ & $\mathbf{3 , 7 2 4 , 1 0 9}$ & $\mathbf{3 , 7 9 9 , 2 1 1}$ \\
\hline \multirow{2}{*}{1.14} & Overset & - & - & 308,390 & 858,515 & 874,993 \\
& Background & - & - & $3,541,852$ & $2,646,615$ & $2,689,829$ \\
& Total & $\mathbf{3 , 2 3 0 , 9 0 6}$ & $\mathbf{3 , 2 5 3 , 5 9 2}$ & $\mathbf{3 , 8 5 0 , 2 4 2}$ & $\mathbf{3 , 5 0 5 , 1 3 0}$ & $\mathbf{3 , 5 6 4 , 8 2 2}$ \\
\hline 1.10 & Overset & - & - & 308,390 & 780,805 & $\mathbf{7 9 5 , 7 9 1}$ \\
& Background & - & - & 3475,296 & $2,663,972$ & $2,697,156$ \\
& Total & $\mathbf{3 , 1 6 9 , 7 4 2}$ & $\mathbf{3 , 1 9 3 , 9 7 2}$ & $\mathbf{3 , 7 8 3 , 6 8 6}$ & $\mathbf{3 , 4 4 4 , 7 7 7}$ & $\mathbf{3 , 4 9 2 , 9 4 7}$ \\
\hline
\end{tabular}

\section{Computation Results}

The predicted squat from each modelling technique is expressed in the form of AP and FP sinkages. experiment is self-propelled. 


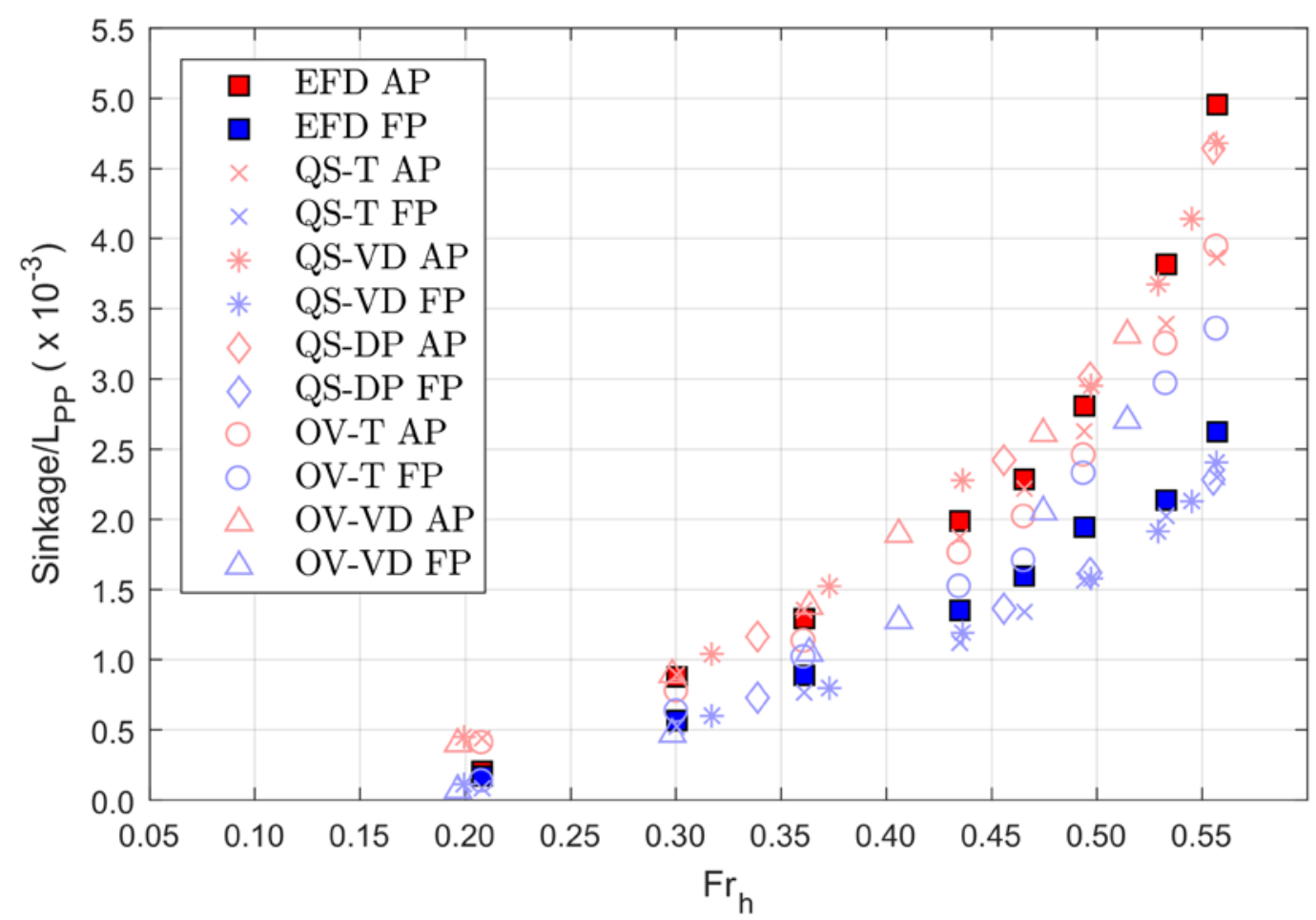

Figure 6: AP \& FP sinkage predictions comparison for $\mathrm{h} / \mathrm{T}=1.23$.

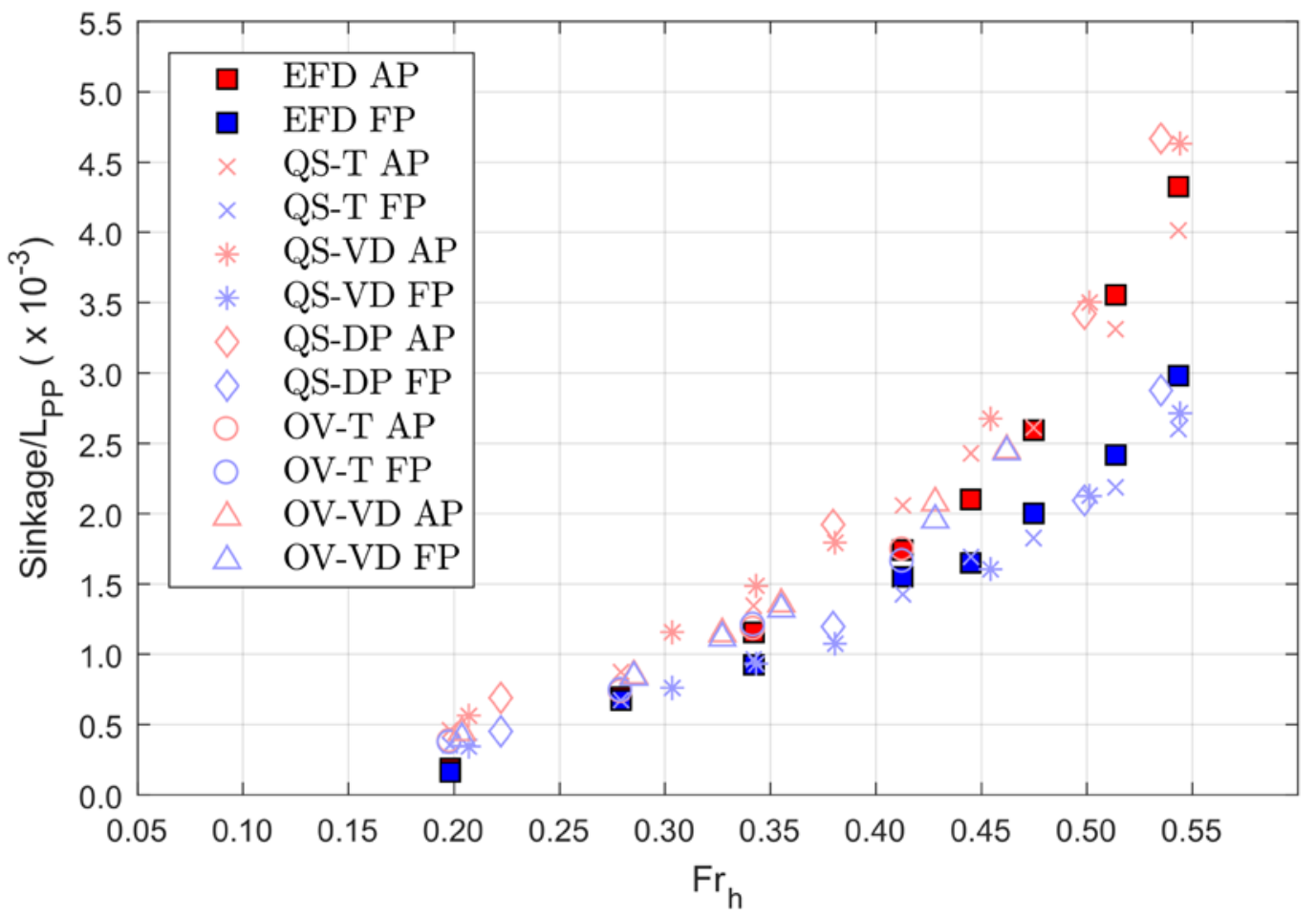

Figure 7: AP \& FP sinkage predictions comparison for $\mathrm{h} / \mathrm{T}=1.14$. 


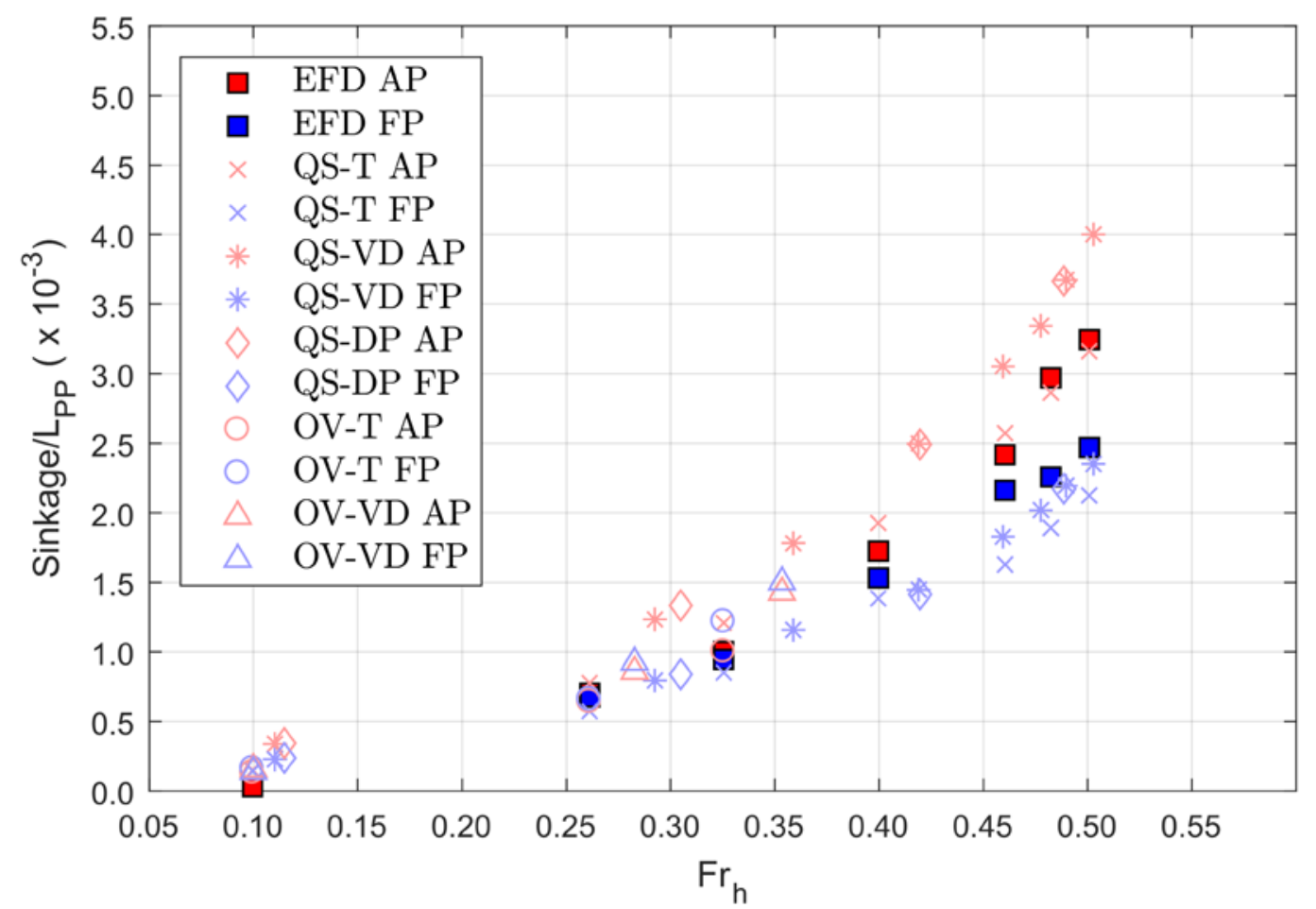

Figure 8: AP \& FP sinkage predictions comparison for $\mathrm{h} / \mathrm{T}=1.10$.

\subsection{Modelling Technique Comparison}

A general overview of the computation requirements and limitations of each modelling technique are tabulated in Table 9. Firstly, each modelling technique has varying processing power and time requirements, and as expected, the QS methods are significantly faster in terms of computational time than OV methods. QS methods are able to converge approximately 4 times faster than OV methods while using $44 \%$ less number of processors. In addition, self-propelled methods by virtual disc body force method require approximately $6 \%$ more computation time than towed methods. In contrast, the self-propelled method by discretised propeller (QS-DP) requires 290\% more computation time while using 50\% more processors than the towed variant (QS-T). Overall, OV-VD is the most computationally intensive followed by OV-T, QS-DP, QS-VD while the most computationally economical method is the QS-T.

In regards to range of applicable speeds, it should be noted that some of the modelling techniques are unable to converge for all the cases investigated in this study. The convergence issue is evident in both 
OV methods especially in shallower cases. At $h / T=1.23$, convergence is achieved for the whole speed range for OV-T but OV-VD could only converge for $\mathrm{Fr}_{\mathrm{h}}<0.52$. At $h / T=1.14$, the convergence limit of OV-T and OV-VD becomes $\mathrm{Fr}_{\mathrm{h}}<0.42$ and $\mathrm{Fr}_{\mathrm{h}}<0.47$, respectively and this limit further reduces for the case of $h / T=1.10$ to $\mathrm{Fr}_{\mathrm{h}}<0.33$ and $\mathrm{Fr}_{\mathrm{h}}<0.36$, respectively. The convergence issue of the $\mathrm{OV}$ methods are attributed to the limitation of enabling DFBI trim and sinkage where there must be sufficient grids (space) in the UKC for the hull to gradually trim and sink in an oscillatory manner until it reaches a dynamic equilibrium irrespective of the ramp time allocated. Convergence cannot be achieved if the initial oscillatory motion causes the hull to contact the bottom boundary. The higher the speed of travel, the greater the initial oscillatory motion becomes and therefore the highest speed that the OV methods can achieve convergence reduces as the UKC decreases (shallower water). The OVVD method generally has a higher convergence limit than OV-T because the build-up in speed for the OV-VD method is due to the force imparted by the virtual disc, which is more gradual and hence the initial oscillatory motion has lower magnitude. However, all QS methods do not have such convergence issues.

Table 9: Summary of the computation requirements and limitations of each modelling technique.

\begin{tabular}{|l|l|l|l|l|l|}
\hline Technique Name & QS-T & QS-VD & QS-DP & OV-T & OV-VD \\
\hline \multicolumn{7}{|c|}{ Simulation Details } \\
\hline Total Time (hrs) & 35 & 37 & 136 & $144-160$ & $150-168$ \\
CPU Time per Time Step (s) & 7 & 7.4 & 27.2 & 30.4 & 31.8 \\
Processors & 104 & 104 & 156 & 182 & 182 \\
\hline \multicolumn{7}{|c|}{ Max Attainable Speed Range } \\
\hline h/T = 1.23 & $\mathrm{Fr}_{\mathrm{h}}=0.56$ & $\mathrm{Fr}_{\mathrm{h}}=0.56$ & $\mathrm{Fr}_{\mathrm{h}}=0.56$ & $\mathrm{Fr}_{\mathrm{h}}=0.56$ & $\mathrm{Fr}_{\mathrm{h}}<0.52$ \\
$\mathrm{~h} / \mathrm{T}=1.14$ & $\mathrm{Fr}_{\mathrm{h}}=0.54$ & $\mathrm{Fr}_{\mathrm{h}}=0.54$ & $\mathrm{Fr}_{\mathrm{h}}=0.54$ & $\mathrm{Fr}_{\mathrm{h}}<0.42$ & $\mathrm{Fr}_{\mathrm{h}}<0.47$ \\
$\mathrm{~h} / \mathrm{T}=1.10$ & $\mathrm{Fr}_{\mathrm{h}}=0.50$ & $\mathrm{Fr}_{\mathrm{h}}=0.50$ & $\mathrm{Fr}_{\mathrm{h}}=0.50$ & $\mathrm{Fr}_{\mathrm{h}}<0.33$ & $\mathrm{Fr}_{\mathrm{h}}<0.36$ \\
\hline
\end{tabular}

In order to assess the accuracy of each technique quantitatively, the difference of AP and FP sinkage predictions relative to benchmark squat data are expressed as a percentage of the maximum benchmark AP and FP squat data for each respective $h / T$ case. Boxplots of the said relative difference for each method for $h / T=1.23, h / T=1.14$ and $h / T=1.10$ are as depicted in Figure 9, Figure 10 and Figure 11 
respectively. The upper and lower edge of the blue box in the boxplots represent the $25^{\text {th }}$ and $75^{\text {th }}$ percentiles. The whiskers indicate variability outside the upper and lower quartiles while the red crosses represent the outliers. Note that the medians are not depicted in these boxplots but the means are shown instead as blue crosses as they are more relevant for such comparisons.

The boxplots show that the QS-T method has low average relative difference at the deepest case but exhibits noticeable dispersion and the maximum AP sinkage underestimation is as low as $22 \%$ at the highest speed (represented as the outlier). However, as $h / T$ decreases, the QS-T method exhibits reduced dispersion and has the best correlation in the shallowest case.

On the contrary, the QS-VD method has excellent correlation in the deepest case but tends to overestimate AP sinkage as $h / T$ decreases which may be due to the inability of the QS methods to resolve non-linearities in forces and moments. The AP sinkage predicted by the QS-VD for the case of $h / T=1.10$ is overestimated on average by $15.7 \%$ and is therefore highly unreliable for this $h / T$. Nonetheless, the QS-VD has reasonable dispersion for cases other than $h / T=1.10$. Generally, the trend of the QS-VD method results has no significant difference from that of the QS-DP. The only key difference is that the QS-DP method appears to have reduced dispersion due to the smaller number of data points in comparison to QS-VD. Hence, if given the same number of data points, the QS-DP method is expected to have almost identical boxplots to that of the QS-VD.

Both OV-T and OV-VD methods predicted AP sinkage with reasonable accuracy for all three $h / T$ cases for $F r_{h}<0.52$. Nonetheless, it is evident from Figure 6, Figure 7 and Figure 8 that the predicted AP and FP sinkage are relatively similar in magnitude compared to that of the benchmark data which implies that the OV methods underpredicted trim. For instance, for $h / T=1.23$, the OV-T and OV-VD methods underpredicted trim by $75 \%$ and $63 \%$ at their respective highest achievable speed whereas trim is barely noticeable for $h / T=1.14$ and $h / T=1.10$. Hence, the OV-VD method has relatively large average error and dispersion for FP sinkage predictions at $h / T=1.23$ and 1.14. At $h / T=1.10$, the OV-VD method appears to have highly favourable average error and dispersion but this is due to the low speed range applicability of the OV-VD method where trim is not significant. Similarly, the OV-T method has large 

for shallower cases appear favourable due to its limited speed range applicability.

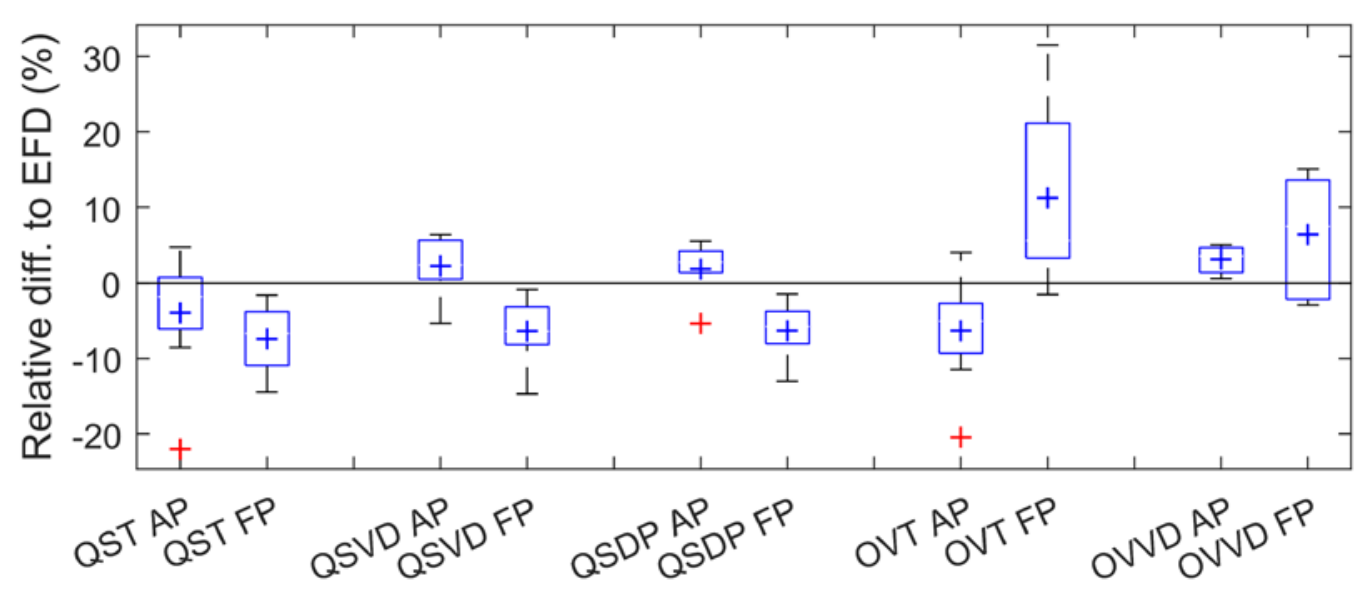

356 Figure 9: Boxplot of the relative difference of each CFD method with respect to the squat at AP and 357 FP from benchmark squat experiment data for case of $\mathrm{h} / \mathrm{T}=1.23$.

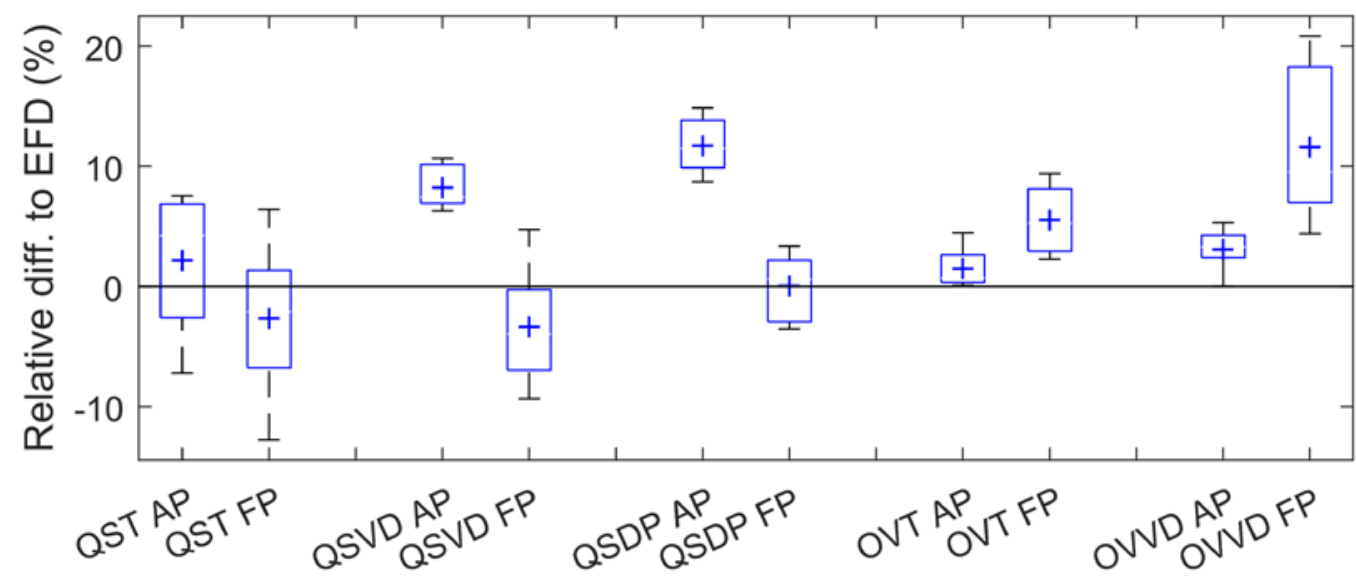

359 Figure 10: Boxplot of the relative difference of each CFD method with respect to the squat at AP and 360 FP from benchmark squat experiment data for case of $\mathrm{h} / \mathrm{T}=1.14$. 


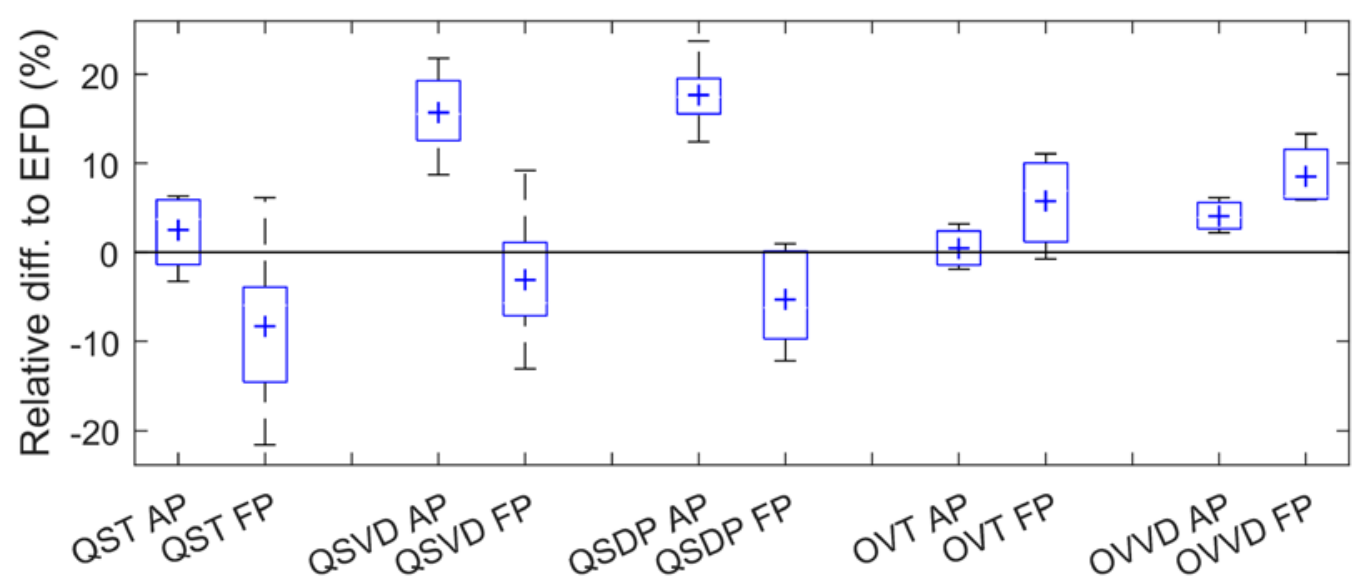

Figure 11: Boxplot of the relative difference of each CFD method with respect to the squat at AP and FP from benchmark squat experiment data for case of $h / T=1.10$.

\subsection{Self-propulsion Effect}

In this section, emphasis will be only on AP sinkage since the maximum sinkage and hence the likely contact region during grounding in this investigation is on the AP of the vessel. As observable from Figure 6, Figure 7 and Figure 8 there exist noticeable differences between the predictions from towed methods to that of the self-propelled methods for all three $h / T$ cases.

At $h / T=1.23$, the AP sinkage predicted by all 5 methods are fairly similar for $\mathrm{Fr}_{\mathrm{h}}<0.3$. Nonetheless, when $\mathrm{Fr}_{\mathrm{h}}>0.3$, it can be seen that the AP sinkage predictions by the self-propelled methods begin to deviate from that of the towed methods. At the highest speed (0.56 $\mathrm{Fr}_{\mathrm{h}}$ ), the towed method, QS-T, underestimated AP sinkage by approximately $17 \%$ with respect to both self-propelled methods; QS-VD and QS-DP. Similarly, for the case of $h / T=1.14$, obvious deviations in AP sinkage prediction can be observed between the towed methods and self-propelled methods at higher speeds. At the highest speed (0.54 $\mathrm{Fr}_{\mathrm{h}}$ ), the towed method, QS-T, underestimated AP sinkage by approximately $14 \%$ relative to that of the self-propelled methods; QS-VD and QS-DP. For $h / T=1.10$, the relative difference at the highest speed $(0.50 \mathrm{Fr} h)$ is $24 \%$. Hence, the effect of self-propulsion on container ship squat is indeed significant especially at higher speeds.

Figure 12 compares the flow velocity at the stern region between the QS-T, QS-VD and QS-DP predictions for the case of $h / T=1.23$ at $0.56 \mathrm{Fr}_{\mathrm{h}}$. It can be observed that the flow in the stern region 
without propeller (Figure 12(a)) is relatively simple whereas that of the QS-VD (Figure 12(b)) and QS-

DP (Figure 12(c)) have more complex and greater flow movement due to propeller action, which results

in lower pressure and hence more stern-down trim for the QS-VD and QS-DP predictions. However, it

is of interest to note that the wake pattern predicted by the QS-VD method differs from that of the QS-

DP and also that the magnitude of the flow velocity is observably smaller for the QS-VD but the overall predicted sinkage and trim by both QS-VD and QS-DP methods are still almost identical. This may imply that the propeller generated trim moment about the LCF has a more significant impact on squat than the magnitude of the propeller wake flow velocity. Regardless, it is clear that self-propulsion effect must be accounted for in the prediction of container ship squat, which can be modelled with sufficient accuracy by means of either virtual disc body-force propulsion method or full discretisation of the propeller.
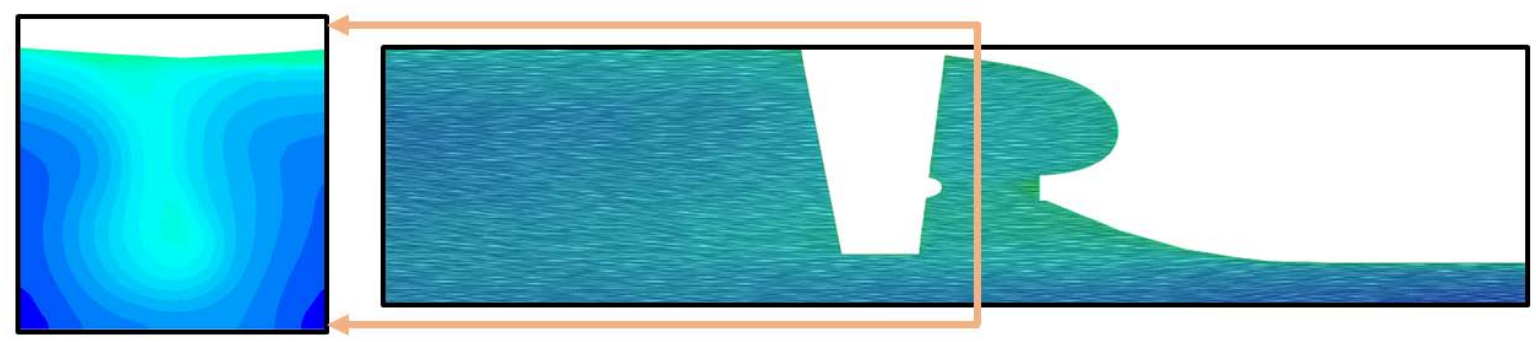

(a)
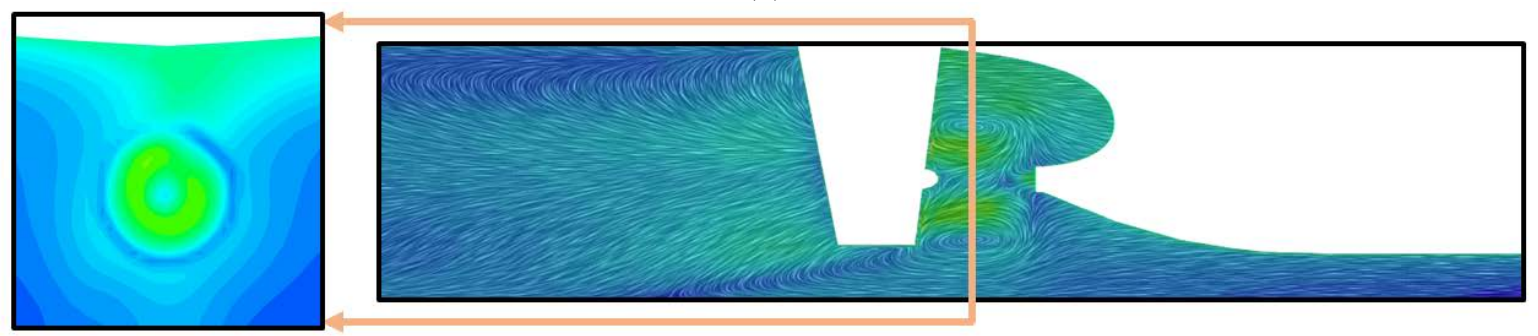

(b)

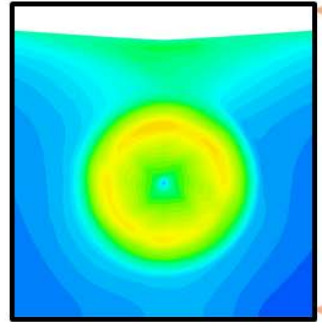

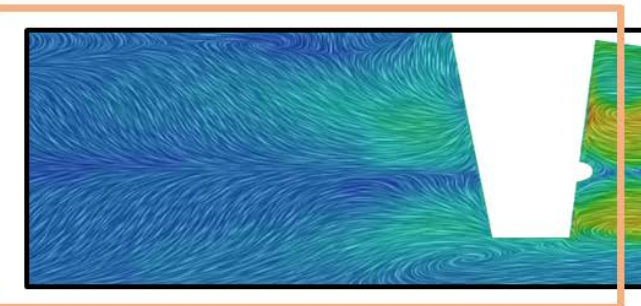

(c)
0.000
0.400
0.800
1.20

\section{.000}

city $(\mathrm{m} / \mathrm{s})$
1.60

2.00

2.40 2.80 
Figure 12: Comparison of the stern region flow velocity (right) and the wake pattern at 0.49 Lpp aft of midships (left) predicted by QS-T (a), QS-VD (b) and QS-DP (c) for h/T = 1.23 at $0.56 \mathrm{Fr}_{\mathrm{h}}$.

Having compared the performance of each method in the previous section and evaluating the effect of self-propulsion, a decision can be made to determine the most suitable modelling technique for the cases tested. Based on the performance comparisons conducted, the QS methods are preferable over OV methods since QS methods are able to converge relatively quickly, have no apparent limits of applicability and are also able to predict trim better. As mentioned earlier, propeller effects are significant at relatively high speeds and thus QS-VD and QS-DP methods are the more favourable methods. However, since the QS-DP method is more resource intensive while producing predictions which have insignificant difference from that of the QS-VD, the QS-VD is the most favourable method. Regardless, it should be noted that the QS-VD (as well as QS-DP) method is not recommended for extremely shallow cases i.e. $h / T<1.14$ as the QS-T method is more accurate for the cases studied. Future studies will investigate whether the QS-VD and QS-T methods are still sufficiently accurate for different container ship hulls. It is also suggested that when implementing the QS method in future studies the predicted sinkage and trim for a previous slower speed can be used as an initial guess as this may be able to partially resolve non-linearities in forces and moments.

\section{Concluding Remarks}

A numerical investigation on container ship squat has been conducted on the DTC container ship model using 5 different techniques based on URANS computations. In the investigation, both quasi-static (QS) and dynamic overset (OV) modelling approaches have been investigated. In addition, different modes of propulsion have also been investigated i.e. the hull is either towed or self-propelled where two propeller modelling methods are compared; virtual disc body-force propulsion and fully discretised propeller. The numerical predictions were compared to benchmark model scale experiment data for the DTC container ship for three different water depth to draft ratios $(h / T): 1.23,1.14$ and 1.10.

From the investigation it is observed that the QS methods are more advantageous than OV methods as the QS methods converge relatively quickly, have no apparent limits for hull speed range and can predict 
trim better. However, it should be noted that the QS methods with only one iteration are unable to resolve non-linearities in forces and moments as the hull squats. In regards to accuracy, self-propelled QS methods are capable of yielding accurate squat predictions except for the shallowest case tested with $h / T=1.10$ which is likely due to the increasing non-linearities in forces and moments. In addition, comparison of the AP sinkage predictions between towed methods and self-propelled methods reveals obvious deviations which implies that the propeller effect is significant particularly at higher speed ranges. Comparisons between the virtual disc propeller modelling technique (QS-VD) and discretised propeller modelling technique (QS-DP) shows no significant difference in the predicted squat. Additionally, the virtual disc model is less computationally intensive than the discretised propeller model. Hence, given that the QS methods are more advantageous and the virtual disc propeller model is as accurate as the discretised propeller model while being more economical, it can be concluded that the QS-VD method is the most favourable option among the modelling techniques investigated in this study. However, the towed quasi-static squat model (QS-T) is recommended for extremely shallow cases where $h / T<1.14$. Future studies will investigate whether the QS-VD and QS-T methods are still valid for different container ship hulls. It is also recommended that when implementing QS methods in future studies, the predicted sinkage and trim for a previous slower speed can be used as an initial guess as this may be able to partially resolve the non-linearities in forces and moments.

\section{Data Availability Statement}

436 The hull and propeller CAD model used in this study are available from the corresponding author by 437 request.

\section{Acknowledgements}

439 The authors acknowledge the funding and resources provided by NCMEH Australian Maritime College, 440 University of Tasmania and the Tasmanian Partnership for Advanced Computing for the HPC for 441 performing the computations. 
References

BARRASS, C. 1979. "The phenomena of ship squat." International Shipbuilding Progress, 26.

BECK, R. F., NEWMAN, J. N. \& TUCK, E. O. 1974. "Hydrodynamic forces on ships in dredged channels." Journal of Ship Research, 19(03), 166-171.

CD-ADAPCO 2014. User guide STAR-CCM+ Version 9.0.6.

CONG, L. \& HSIUNG, C. 1991. "Computing wave resistance, wave profile, sinkage and trim of transom stern ships." Proceedings of the Third International Conference on Computer Aided Design, Maunfacture and Operation in the Marine and Offshore Industries (CADMO 91), 99-112. Key Biscayne.

CONSTANTINE, T. 1960. "On the movement of ships in restricted waterways." Journal of Fluid Mechanics, 9, 247-256.

DAND, I. \& FERGUSON, A. 1973. "The squat of full ships in shallow water." National Physical Lab Teddington (England) Ship Div.

DELEFORTRIE, G., VANTORRE, M., ElOOT, K., VERWILligeN, J. \& LATAIRE, E. 2010. "Squat prediction in muddy navigation areas." Ocean Engineering, 37, 1464-1476.

DENG, G., GUILMINEAU, E., LEROYER, A., QUEUTEY, P., VISONNEAU, M. \& WACKERS, J. 2014. "Simulation of container ship in shallow water at model scale and full scale." Proceedings of the 3rd National CFD Workshop for Ship and Offshore Engineering, Dalian, China.

DUFFY, J. \& RENILSON, M. 2000. "An investigation into the effect of propulsion on ship squat." Oceanic Engineering International, 4, 1-12.

DUFFY, J. T. 2008. Modelling of ship-bank interaction and ship squat for ship-handling simulation. University of Tasmania.

ELOOT, K., VERWILLIGEN, J. \& VANTORRE, M. 2008. "An overview of squat measurements for container ships in restricted water." International conference on safety and operations in canals and waterways (SOCW) 2008, 106-116. Glasgow, UK.

FUEHRER, M. \& RÖMISCH, K. 1977. "Effects of modern ship traffic on islands and ocean waterways and their structures." 24th International Navigation Congress, PIANC, 236-244. Leningrad, USSR.

GOURLAY, T., LATAIRE, E. \& DELEFORTRIE, G. 2016. "Application of potential flow methods to ship squat in different canal widths." 4th MASHCON, 146-155. Bundesanstalt für Wasserbau, Germany.

GOURLAY, T. P., HA, J. H., MUCHA, P. \& ULICZKA, K. 2015. "Sinkage and trim of modern container ships in shallow water." Proceedings of the Australasian Coasts \& Ports Conference 2015, Auckland, New Zealand.

JACHOWSKI, J. 2008. "Assessment of ship squat in shallow water using CFD." Archives of Civil and Mechanical Engineering, 8, 27-36. 
LATAIRE, E., VANTORRE, M. \& DELEFORTRIE, G. 2012. "A prediction method for squat in restricted and unrestricted rectangular fairways." Ocean Engineering, 55, 71-80.

MUCHA, P., EL MOCTAR, O. \& BÖTTNER, C.-U. 2014. "PreSquat-Workshop on numerical prediction of ship squat in restricted waters." Ship Technology Research, 61, 162-165.

NAGHDI, P. \& RUBIN, M. 1984. "On the squat of a ship." Journal of Ship Research, 28, 107-117.

SHEVCHUK, I., BÖTTNER, C.-U. \& KORNEV, N. 2016. "Numerical Analysis of the Flow in the Gap Between the Ship Hull and the Fairway Bottom in Extremely Shallow Water." 4th MASHCONInternational Conference on Ship Manoeuvring in Shallow and Confined Water, 37-42. Hamburg, Germany.

STERN, F., WILSON, R. V., COLEMAN, H. W. \& PATERSON, E. G. 2001. "Comprehensive approach to verification and validation of CFD simulations-part 1: methodology and procedures." Journal of fluids engineering, 123, 793-802.

TEZDOGAN, T., DEMIREL, Y. K., KELLETT, P., KHORASANCHI, M., INCECIK, A. \& TURAN, O. 2015. "Fullscale unsteady RANS CFD simulations of ship behaviour and performance in head seas due to slow steaming." Ocean Engineering, 97, 186-206.

TEZDOGAN, T., INCECIK, A. \& TURAN, O. 2016. "A numerical investigation of the squat and resistance of ships advancing through a canal using CFD." Journal of Marine Science and Technology, 21, 86-101.

TUCK, E. 1966. "Shallow-water flows past slender bodies." Journal of Fluid Mechanics, 26, 81-95.

TUCK, E. 1973. "Sinkage and trim in shallow water of finite width." Schiffstechnik, 14.

WILSON, R. V., STERN, F., COLEMAN, H. W. \& PATERSON, E. G. 2001. "Comprehensive approach to verification and validation of CFD simulations-Part 2: Application for RANS simulation of a cargo/container ship." Journal of fluids engineering, 123, 803-810.

YAO, J.-X. \& ZOU, Z.-J. 2010. "Calculation of ship squat in restricted waterways by using a 3D panel method." Journal of Hydrodynamics, Ser. B, 22, 489-494.

ZHANG, Z.-H., DENG, H. \& WANG, C. 2015. "Analytical models of hydrodynamic pressure field causing by a moving ship in restricted waterways." Ocean Engineering, 108, 563-570. 